\title{
UDC 621.039.672 \\ DEVELOPMENT AND APPLICATIONS OF HINEG HIGH INTENSITY NEUTRON SOURCES
}

(short message)

Yican $W u$

Institute of Nuclear Energy Safety Technology, FDS Team, Chinese Academy of Sciences, Hefei, Anhui, 230031, China

Neutron sources are the important experimental platforms for the R\&D of advanced nuclear energy and nuclear technology application. The High Intensity Neutron Generator (HINEG) has been developed in China with different missions including neutronics design validation, material \& components irradiation test, nuclear waste burning and nuclear technology application. HINEG-I has achieved the fusion neutrons with the yield of $6.4 \cdot 10^{12} \mathrm{n} / \mathrm{s}$ at maximum, and has been coupled with the Lead-based Zero Power Critical/Subcritical Reactor named CLEAR-0. Such a facility is actually an accelerator-driven Fusion-Fission Hybrid System. Series of typical experiments have been carried out on HINEG-I, including neutronics and code validation, core physics study of advanced reactors, neutron radiography, neutron detector calibration, neutron biological effects, neutron radiation hardening, and so on. HINEG-II is an accelerator-based neutron source with the yield of $10^{14}$ $10^{15} \mathrm{n} / \mathrm{s}$. It aims to apply to multi-purposes, e.g. neutron capture therapy, isotope production, etc. The design and R\&D for key technologies of HINEG-II are performed on-going. HINEG-III is initially conceived as a GDTbased or accelerator-based neutron source with the intensity of $10^{17}-10^{18} \mathrm{n} / \mathrm{s}$. The objectives of HINEG-III are to conduct test of nuclear materials, components test and reliability data collection of nuclear components, nuclear waste burning test, etc. This contribution presents an overview of the series recent activities.

Key words: neutron source, HINEG, high intensity.

DOI: $10.21517 / 0202-3822-2021-44-2-163-164$

\section{РАЗРАБОТКА И ПРИМЕНЕНИЕ ВЫСОКОИНТЕНСИВНЫХ НЕЙТРОННЫХ ИСТОЧНИКОВ НINЕG}

(краткое сообщение)

\author{
Й. Вy
}

Институт технологии безопасности ядерной энергии, Команда FDS, Китайская академия наук, Хэфэй, Аньхой, 230031, Китай

Источники нейтронов являются важными экспериментальными установками для исследований и разработок в области передовой ядерной энергетики и применения ядерных технологий. Высокоинтенсивный нейтронный генератор (HINEG) был разработан в Китае с различными задачами, включая проверку нейтронно-физических расчётов, испытание под облучением материалов и компонентов, сжигание ядерных отходов и применение ядерных технологий. Ha HINEG-I достигнут максимальный выход термоядерных нейтронов $6,4 \cdot 10^{12}$ н/с. Этот нейтронный источник был соединён со свинцовым критическим/подкритическим реактором нулевой мощности CLEAR-0. Полученная установка является гибридной системой синтеза-деления на основе ускорителя. Ha HINEG-I была проведена серия типичных экспериментов, включая проверку нейтроники и валидацию нейтронного кода, изучение нейтронной физики активной зоны перспективных реакторов, нейтронную радиографию, калибровку нейтронных детекторов, нейтронные биологические эффекты, нейтронное радиационное упрочнение и т.д. HINEG-II источник нейтронов на основе ускорителя с выходом $10^{14}-10^{15}$ н./с. Он предназначен для применения в различных целях, например, в нейтронно-захватной терапии, производстве изотопов и т.д. Проектирование и НИОКР по ключевым технологиям HINEG-II ведутся в постоянном режиме. HINEG-III изначально задумывался как источник нейтронов на основе ГДЛ или ускорителя с интенсивностью $10^{17}-10^{18}$ н./c. Целями HINEG-III являются проведение испытаний ядерных материалов, испытание компонентов и сбор данных о надёжности ядерных компонентов, испытание на сжигание ядерных отходов и т.д. Доклад представляет собой обзор серии недавних работ.

Ключевые слова: источник нейтронов, HINEG, высокая интенсивность.

yican.wu@fds.org.cn 